\title{
Laboratory Investigation for Diabetes Mellitus: Practical Concerns
}

\author{
Viroj Wiwanitkit
}

Wiwanitkit House, Bangkhae, Bangkok 10160, Thailand

\begin{abstract}
Diabetes mellitus (DM) is the most common endocrine problem at present. Based on the concept of good laboratory investigation management, the full overage on quality of these tests are required. In this article, the author hereby discusses on some practical concerns for important laboratory investigations for DM.
\end{abstract}

Keywords: Practice, concern, laboratory, diabetes mellitus.

\section{INTRODUCTION}

Diabetes mellitus (DM) is the most common endocrine problem at present. Millions of world population suffer from DM. The diagnosis of this endocrine disorder bases mainly on laboratory investigations. The fasting plasma glucose (FPG) is the tool for definite diagnosis of DM. In addition, several other laboratory investigations are useful in diagnosis and following up of case with DM. These investigations are of interest.

Based on the concept of good laboratory investigation management, the full overage on quality of these tests are required. In this article, the author hereby discusses on some practical concerns for important laboratory investigations for DM.

\section{FASTING PLASMA GLUCOSE: BASIC BUT IMPORTANT}

FPG is the very basic test for DM. FPG is useful for both diagnosis and following up. This is a basic test but very important. The pre-analytical factor seems to strongly affect the FPG results. The patient preparation must be gently controlled. It is a requirement to verify the fasting stage of the patients. This has to be carefully checked. Some patients might disguise the fact that they ingest or drink something before the blood collection which can result in the aberration of FPG results. For example, a diabetic patient disguised that he drank soft drink before blood collection and his FPG result was very high. After that the physician in charge prescribed for new stronger dosage of anti-diabetic drug and which resulted in bleeding complication due to drug induced thrombocytopenia.

When performing blood collection for FPG, special attention to the patient must be given because some patients might develop severe hypoglycemia which might lead to sudden death on blood collection [1]. There is very high risk for diabetic patients who fast but still use insulin injection. Special concern should be provided for these cases. Focusing on the blood collection tube, sodium fluoride tube, gray stopper (tube), must be selected. However, in case that FPG

*Address correspondence to this author at the Wiwanitkit House, Bangkhae, Bangkok 10160, Thailand; E-mail: wviroj@yahoo.com is coordered with other basic clinical chemistry test, plasma, red stopper (tube) can be used.

\section{CAPILLARY GLUCOSE DETERMINATION: POINT OF CARE TESTING [2-4]}

Capillary glucose determination by glucometer is accepted as a very useful tool in monitoring of DM case. It is accepted as the most widely used point of care testing at present. This can shorten the turnaround time for the laboratory analysis. The bed side test can be done. Also, it is presently widely used in ward and emergency room for fast result and indicated for the scenario that requires prompt decision for management of DM patients (such as the condition that the patient is unconscious which might be due to hypoglycemia or hyperglycemia). In addition, glucometer is already considered as an extended indicator for other purposes. It is presently applied as daily self monitoring tool for diabetic patients. In the past, urine glucose test strip was used for diagnosis but the strip lacks good diagnostic property and detection of sugar in urine usually implies severity of disease. There are many reports on glucometers for capillary glucose determination. These reports usually indicate the concordance between capillary glucose result and standard FPG result. However, it should be noted that results from glucometer are usually lower than that of standard FPG (because of the nature capillary blood sample). In follow-up of the patient, the tools such as FPG or glucometer from which the blood glucose results are obtained should be verified. Comparison between results from different tools must be specially and carefully done.

\section{POSTPRANDIAL GLUCOSE: AN ALTERNATIVE MEASUREMENT FOR BLOOD GLUCOSE}

Sometimes, the FPG test is not feasible hence the postprandial glucose is examined. This might be another alternative clue for diagnosis of DM. One with abnormal high postprandial glucose level needs further verification by FPG test. The postprandial glucose is an actual application of measurement of chemical in blood with the interference (from diet). However, the practical problem in usage of postprandial glucose is the difficulty in setting for accurate cut-off point for the abnormality. For some instance, a control of diet intake and set of time can be useful for application in diagnosis. This is the concept of the glucose 
challenge test and oral glucose tolerance test, which are widely used in obstetrics.

\section{GLYCATED END PRODUCT: TOOL FOR MONITORING FOR DIABETIC COMPLICATION}

Glycated end product is a specific in vivo substance that can be generated in case of poorly controlled DM patients. This is the result from the reaction between proteins in blood (normally hemoglobin or albumin) with excessive glucose in blood. The nature of energy aberration induction of the reaction results in complications of DM [5]. The two main tests on glycated end products are hemoglobin A1C (HbA1C) and fructosamine. $\mathrm{HbA1C}$ gas has a wider range of prediction, upto 3 months, while that of fructosamine is about 1 month. However, HbA1C should be carefully interpreted in setting with high prevalence of hemoglobinopathy because this can deviate the result [6]. In addition to the two quoted tests, glycoalbumin test is also available.

Although glycoalbumin is more reliable than simple fructosamine but the standard method of measurement has not been established yet [7]. In addition, the limitation of usage of glycolalbumin is also expected in diabetic case complicated with protein malnutrition [7].

\section{ORAL GLUCOSE TOLERANCE TEST AND DIABETES IN PREGNANCY}

Oral glucose tolerance test (OGTT) is the main test for diagnosis of DM in pregnancy, which is a specific endocrine disorder in obstetrics. OGTT is generally indicated after positive urine glucose test and glucose challenge test. The main practical concern for OGTT is the preparation of the patient for the test [8]. Hypoglycemia can still be detectable during OGTT [9]. In addition, some pregnant cases might have hyperemesis gravidarum which might lead to abnormal low resulted glucose levels. For collection of blood, the order of collected samples must be gently controlled because alteration of the order can make a total difference in interpretation.

\section{URINE TEST FOR DIABETES: NEW AND OLD}

Urine glucose test is an old classical test for DM. In the past, it was primarily observed that the DM cases usually voided urine contaminated with sugar and this is the basic concept for usage of urine glucose test. However, this test is now out of use due to above mentioned reasons. The new test, microalbuminuria test [10-12], is the determination of microquantity of albumin in urine sample. The positive microalbuminuria means the glomerular pathology which is a common complication of DM. However, positive microalbuminuria can also be detectable in other medical disorders including hypertension, which usually corepresents with DM.

\section{URINE KETONE AND BLOOD KETONE: TOOL FOR DIAGNOSIS OF DIABETIC KETOACIDOSIS [13-15]}

Hyperglycemia is an important complication of DM. An important type of hyperglycemia is diabetic ketoacidosis. The diabetic ketoacidosis is a specific condition with severe hyperglycemia, hyperosmolarity and accumulation of ketone in the body of patients. As ketone can be washed out via respiratory tract, the patients usually present quick respiration. Basic test, plasma glucose determination is useful for diagnosis of increased blood sugar level but it is unable to definitely diagnose ketosis. Also, the electrolyte imbalance and increased BUN can be observed due to hyperosmolarity but they cannot lead to definite diagnosis of diabetic ketoacidosis. The definite diagnosis of ketoacidosis must be based on determination of urine in ketone or ketone in blood. The beta hydroxybutyrate is the specific identified form of ketone in diabetic ketoacidosis.

\section{C-PEPTIDE: A NEW TEST IN DIABETIC MEDICINE}

C-peptide is considered as a new test in diabetic medicine. It is mentioned useful in follow-up of the diabetic complication [16]. It is noted for the good diagnostic property for diabetic neuropathy, one of the most common complications of DM $[17,18]$. Meier et al. concluded that cpeptide was a simple, cost-effective and non-invasive method in the assessment of beta-cell capacity [19]. However, the main limitation for C-peptide test is the rather expensive laboratory cost and availability of the test.

\section{INSULIN TEST: A DIRECT MEASUREMENT OF HORMONE}

Insulin is the major hormone in regulation of blood glucose hemeostasis. The abnormality of insulin physiology is mentioned as an important underlying for DM. The measurement of insulin can be done but it is not practically used in routine diabetic clinic. In addition to simple insulin level measurement, glucose clamp technique (the hyperinsulinemic-euglycemic clamp in assessing in vivo insulin sensitivity and the hyperglycemic clamp in assessing insulin secretion) is also performed to measure insulin secretion and sensitivity $[20,21]$. The principle of this technique is to open the homeostatic feedback loop [20]. This technique is useful for accurate assessment of beta cell function $[20,21]$. For sure, the clamp technique is difficult and should be performed by the experts in diabetes medicine.

\section{REFERENCES}

[1] Wiwanitkit V. Case of sudden death in venipuncture clinic Phlebology 2004; 19: 193

[2] Blake DR, Nathan DM. Point-of-care testing for diabetes. Crit Care Nurs Q 2004; 27: 150-61.

[3] Price CP. Point-of-care testing in diabetes mellitus. Clin Chem Lab Med 2003; 41: 1213-9.

[4] Suzuki M. Present status and tasks for future of point of care testing. Rinsho Byori 2002; 50: 953-7.

[5] Wiwanitkit V. Energy consumption for the formation of hemoglobin A1c: a reappraisal and implication on the poor-control diabetes mellitus patients. J Diabetes Compl 2006; 20: 384-6.

[6] Wiwanitkit V. Problem of using hemoglobin A1C measurement in endemic area of hemoglobinopathy. Prim Care Diabetes 2007; 1 : 173-5.

[7] Matsuyama T. Recent progress in diagnoses of diabetes and its complications. Rinsho Byori 1995; 43: 1235-40.

[8] Theodoraki A, Baldeweg SE. Gestational diabetes mellitus. Br J Hosp Med 2008; 69: 562-7.

[9] Weissman A, Solt I, Zloczower M, Jakobi P. Hypoglycemia during the $100-\mathrm{g}$ oral glucose tolerance test: incidence and perinatal significance. Obstet Gynecol 2005; 105: 1424-8.

[10] Polkinghorne KR. Detection and measurement of urinary protein Curr Opin Nephrol Hypertens 2006; 15: 625-30.

[11] Comper WD, Osicka TM. Detection of urinary albumin. Adv Chronic Kidney Dis 2005; 12: 170-6.

[12] Busby DE, Atkins RC. Detection and measurement of microalbuminuria: a challenge for clinical chemistry. Med Lab Obs 2005; 37: 8-9, 12, 14-6. 
[13] Kearney T, Dang C. Diabetic and endocrine emergencies. Postgrad Med J 2007; 83: 79-86.

[14] Reingardiene D. Diabetic ketoacidosis. Medicina (Kaunas) 2002; 38: 666-72.

[15] Bruyette DS. Diabetic ketoacidosis. Semin Vet Med Surg (Small Anim) 1997; 12: 239-47.

[16] Wahren J, Jörnvall H. C-peptide makes a comeback. Diabetes Metab Res Rev 2003; 19: 345-7.

[17] Sima AA. C-peptide and diabetic neuropathy. Expert Opin Investig Drugs 2003; 12: 1471-88.
[18] Matsuda A. C-peptide in blood. Nippon Rinsho 1998; 56(Suppl 3): 35-9.

[19] Meier C, Ladewig A, Keller U, Berger W. Clinical value of Cpeptide determination. Praxis (Bern 1994) 1997; 86: 1289-95.

[20] Scheen AJ. Evaluation of insulinosecretion and insulinosensitivity. Therapie 2007; 62: 311-8.

[21] Arslanian SA. Clamp techniques in paediatrics: what have we learned? Horm Res 2005; 64(Suppl 3): 16-24.

(C) Viroj Wiwanitkit; Licensee Bentham Open.

This is an open access article licensed under the terms of the Creative Commons Attribution Non-Commercial License (http://creativecommons.org/licenses/by$\mathrm{nc} / 3.0 /$ ) which permits unrestricted, non-commercial use, distribution and reproduction in any medium, provided the work is properly cited. 